\title{
Network Analysis as a Research Methodology in Science Education Research ${ }^{1}$
}

\author{
Jesper Bruun, Robert Evans
}

\begin{abstract}
With three examples, we explore different ways of conceptualizing networks of nodes and links as educationally relevant entities. We show how one can analyse networks as they evolve over time - the dynamics of networks - and how one can model dynamic processes on networks. We also explain how networks can have both visual and mathematical properties that make them tractable as a way of generating knowledge about relational data. We suggest how a theory that emphasizes relational aspects might be developed using networks by providing an example about social networks, where we explain the generation of the theory-like structures rules of interaction, which are meant to have explanatory power. Likewise, we make interpretations of student discussions that are shaped in part by the algorithm we use to create discussion maps as well as by the changes we make to the transcript. The article illustrates how in a teacher-student dialogue, the structure of the network shapes the way we comprehend and talk about discussion networks. All these are dependent on the relational character of networks and would not be the same without that perspective. In other words, the theoretical positions we develop are shaped by the nature of networks. We conclude the article by discussing three developments, which need to occur to realize the potential of using network analysis in educational research.
\end{abstract}

Keywords: network analysis, dynamic, relational, methodological tool.

\section{INTRODUCTION}

Teaching-learning situations in general are complex processes that involve relationships between different entities at different levels (see e.g. Davis \& Sumara, 2006; Artigue \& Winsløw, 2010). For example, at the classroom level, students develop relationships both with each other and with the teacher. These relationships have meaning both on social and academic levels. On the level of the individual mind, we as researchers imagine and investigate relationships between concepts, attitudes, and beliefs (e.g. Deci \& Ryan, 2011; diSessa, 2002; Fauconnier \& Turner, 2002). On the level of group discussion, bits of discourse are related through the way they are put to use in

\footnotetext{
${ }^{1}$ This article is based on a presentation given at XXIV. Annual Conference of the Czech Pedagogical Research Association, Networking in Pedagogical Research, September 15-16, 2016.
} 
relation to each other (e.g. Lemke, 1990; Wood \& Kroger, 2000; Gregoric, Planinsic, \& Etkina, 2017). On the level of a lesson, different activities are related through sequencing and learning goals (e.g. Clement, 1993; Lijnse \& Klaassen, 2004; Brewe, 2008). The common denominator here is relations. Our premise for this article is that a methodology that can take into account the inherently relational nature of teachinglearning situations is worth exploring. Network analysis is such a methodology, and this article can be seen as an exploration of network analysis as a methodological tool based on distinct empirical studies, which serve as examples.

In recent years, network analysis has been used to find social and cognitive patterns in a variety of settings (see e.g. Bruun, 2016). In general, one can conceive of a network as a set of entities that are linked via a set of connections. We refer to the entities as nodes and to the connections as links. A network can consist of nodes and links that represent different kinds of connections. For example, in social network analysis, nodes will often represent a person, and links might represent friendships or collaborations (see e.g. Barabási, 2016). In linguistic network analysis (Masucci \& Rodgers, 2006), nodes represent words and links are drawn, for example, if a word appears directly after another word. However, nodes can also represent actions. For example, Shaffer et al. (2009) map different actions that students take during an instructional sequence by saying that if two actions appear within a unit of analysis they are connected. Their unit of analyses are broad and include physical actions but also written and verbal actions.

In principle, what may constitute a network is almost only limited by imagination. However, social network analysis is by far the most common way of applying network analysis in science education (see e.g. Macfadyen \& Dawson, 2010; Daly, 2010; Brewe, Kramer, \& Sawtelle, 2012; Goertzen, Brewe, \& Kramer, 2013; Bruun \& Brewe, 2013; Bruun \& Bearden, 2014; Grunspan, Wiggins, \& Goodreau, 2014; BuchenrothMartin, DiMartino \& Martin, 2017). We find the area important and in the following section, we will illustrate some of the key affordances of networks using social networks. However, we also wish to explore beyond social networks, because we find network analysis as a framework to be versatile. Thus, in this text we will give two examples on how to employ networks in science education research and one example of integrated analysis. In the first example, we show how a formalized student-teacher dialogue can be coded to find patterns in dialogical actions. In the second example, we show how a group discussion can be mapped bottom up starting from a transcript of the words uttered by students in the discussion. Both methods utilize network analysis as a way of analyzing data, but they have different emphases and scopes. In the third example, we provide a novel way of integrating network analysis with qualitative discourse analysis to develop a bottom-up systematic approach to generating themes in data. We will discuss these cases further in the concluding section.

Each of the three examples is to be seen as a vignette in the following sense: We have in- 
tentionally left out detailed information that is relevant for a full comprehension of each study. We have made this choice in order to show the breadth of applicability for network analysis in science education. However, each example is built on either published work or work that has been submitted to international peer-reviewed journals. We refer interested readers to this work in each section.

\section{Three Cases of Network}

\section{ANALYSIS}

\section{Affordances of Social Networks}

In our view, networks have both visual and mathematical affordances. Figure 1 shows two social networks (from Bruun, 2012). Both networks depict a single Danish upper secondary physics class, and each link represents an interaction with a student as perceived by another student. All interactions in these networks revolve around the processes of problem solving in physics. Shades of grey in nodes represent biological gender: the dark grey nodes are male students, and the light grey nodes represent female students. The networks were created based on student answers to the question: "With whom do you remember having communicated about solving physics problems in the preceding week?". We made explicit to students that we also included laboratory work in our

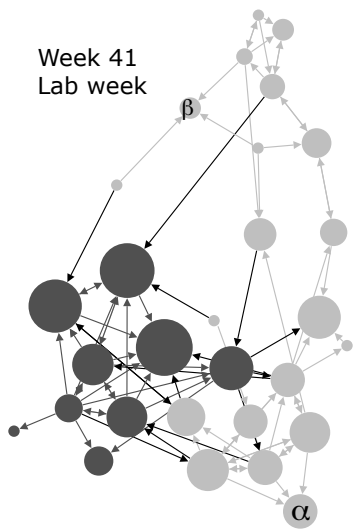

(A)

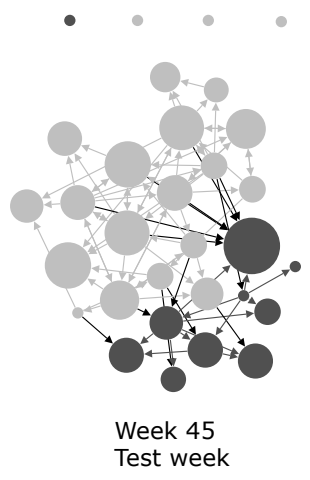

(B)
Target entropy variation

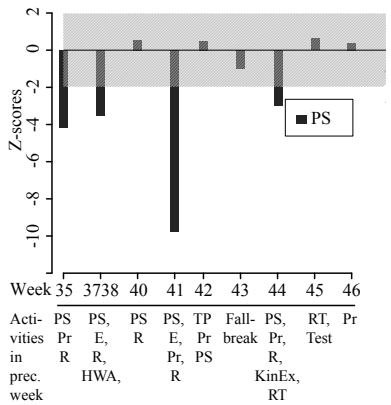

(C)

Figure $1(\mathbf{A}+\mathbf{B})$ Social networks of self-reported student interactions about problem solving Week 41 contains 29 students (20 female and 9 male) and 85 interactions, while Week 45 contains 31 students ( 21 female and 10 male) and 83 interactions. Dark grey nodes represent male students, while light grey represent females. Black arrows represent between-gender interactions, while light and dark grey arrows represent within-gender interactions. The size of nodes is proportional to that node's target entropy. (C) Variation of total target entropy over time for the same class as compared with random expectation. (Adapted from Bruun, 2012.) 
definition of problem solving. Students could pick their classmates from a roster. Students were asked this question at different times during a fall semester. See Bruun (2012, pp. 85-130) for the full rationale and methodological development. The two networks depict the networks for Week 41 and Week 45 and have a comparable number of nodes and links.

We used Gephi software (Bastian, Heymann, \& Jacomy, 2009) to draw the networks using an algorithm that treats nodes as 'particles' and connections as 'springs'. The algorithm continuously updates calculations on-screen to find a stable configuration. The result is that groups that share many connections tend to cluster together when using this particular algorithm. Visually, this can then aid researchers in generating hypotheses about the class. For example, it seems that in both weeks male students (darker grey nodes) seem to cluster together in this class.

We can also see that the network seems much more tightly bound in Week 45 as compared to Week 41. This is interesting when we compare the network with external information about the kind of teaching that took place in the two weeks. In Week 41, students mainly worked on a laboratory exercise, while in Week 45 they were preparing for a test.

A final visual aspect which we draw attention to here is the number of links between males and females. In both weeks, it seems that most links occur between the same biological genders (light and dark grey arrows). Also, the majority of links between different biological genders (black arrows) go from females to males. The effect is much more pronounced in Week 45, where no male has indicated communicating with a female student.

Mathematically, we can verify this by counting the links. The results are summarized in Table 1. By far the majority of links in both weeks are within genders, and it is striking that in Week 45, the amount of links reported by males drops by more than $50 \%$, with no reported interactions from males to females. In contrast, females report more links both to other females and to males. Whether this change in behavior stems from development of class culture over time or because of the different teaching activities that seemed to dominate the two weeks is unknown.

We can also extend the mathematical analysis. Notice that the nodes in each network in Figure 1 have different sizes. The sizes are proportional to a measure called the target entropy (Sneppen, Trusina, \& Rosvall, 2005; Bruun \& Brewe, 2013). Bruun and Brewe (2013) showed that this measure is related to academic performance. The measure presupposes a crude underlying model where every node sends

Table 1 Summary of links within and between genders for the two networks shown in Figure

\begin{tabular}{|l|c|c|c|c|c|}
\hline & Total & Female-female & Male-male & Female-male & Male-female \\
\hline Week 41 & 85 & 44 & 26 & 10 & 5 \\
\hline Week 45 & 83 & 57 & 13 & 13 & 0 \\
\hline
\end{tabular}


out messages continuously through all of its links. If we imagine that we stand at a particular node, the question is then how easy it is to predict from where the next message will come. For example, if a node has only one incoming connection, then a message will always come from that connection. The target entropy for such a node is 0 . If a node has more than one incoming connection, the target entropy is larger than 0 . However, the exact value depends on the wiring of the network. In Figure 1, for example, notice that the node labeled $\alpha$ has three incoming connections, but is bigger than the node $\beta$, which also has three incoming connections. The reason is that two of $\beta$ 's three connections only have outgoing connections, so they do not receive and pass on any messages. However $\beta$ 's third connection does. In contrast, all of $\alpha$ 's connections relay many messages, and thus it is difficult to predict from where the next message will come.

While individual differences in target entropy can be worthwhile to examine, interesting patterns can be found by comparing types of nodes (students) on a larger scale. This can be done both visually and mathematically. Visually, the nodes that represent males in Week 41 are larger on average than the nodes that represent females. In Week 45 the sizes are more equally distributed. We believe that this is related to a change in the way students organized themselves, either because of time or as a response to a changing teachinglearning context.

We are also able to find some interesting regularities that emerge over time. Since data was collected throughout a semester, we are not limited to comparing Week 41 and 45 but can also compare weeks on a longer time scale using target entropy. For each week, we sum up the target entropy of all nodes. Then, also for each week, we create randomized versions of the network for that week. We switch connections so that every student keeps the number of incoming and outgoing connections they have, but we change to whom they are connected (Maslov, Sneppen, \& Zaliznyak, 2004). Then we calculate the total target entropy for the randomized network. We do this for a large number of randomized versions of each week's network. Finally, we compare the total target entropy of a network with the average total target entropy of the randomized networks. Figure $1 \mathrm{C}$ shows the results of this procedure for each week, where we couple these results with the corresponding teaching activities (obtained from teacher $\operatorname{logs}$ ). Notice that each dip in target entropy coincides with laboratory exercises.

Bearing in mind that target entropy is a model of information sharing, we can use these patterns to extrapolate candidate rules of interactions (Forsman, Moll, \& Linder, 2014) for this class of physics students. For example, when doing experimental work, these students are unlikely to share their experiences outside of a small group. If this is true and if knowledge sharing is important, then work has to be done by the teacher to ensure students engage with each other and share their different experiences.

This example shows how visual and mathematical aspects of networks can be 
utilized to study social learning patterns in science education. It relied on student self-reports of their interactions, and did not contain detailed information about these interactions. The next example shows a more fine-grained mapping of an interaction; a dialogue between students and a teacher.

\section{Mapping Teacher-Student Dialogues}

This second example maps student and teacher verbal interactions in two different classrooms to reveal patterns and relationships not easily deduced from transcripts. Our assumption is that a dialogue has quality when it gives students the possibility for formatively and summatively assessing their own competence. To investigate

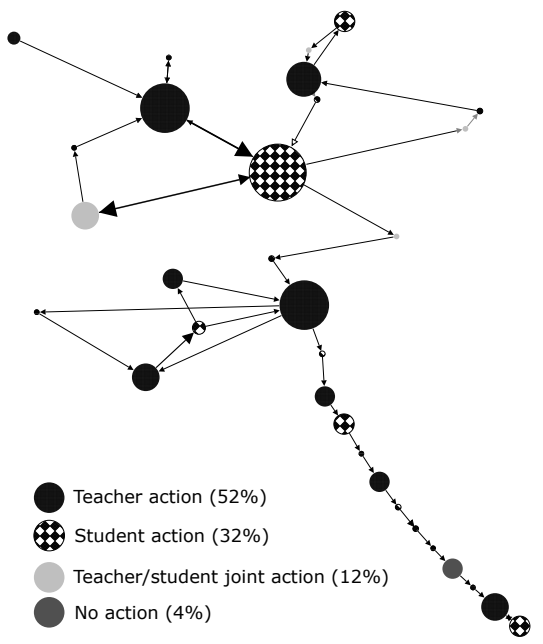

dialogues, video recordings of studentteacher dialogues were coded based on a framework developed to capture dialogical interactions. See Dolin, Bruun, Nielsen, Jensen, \& Niemenen (2018) for the full rationale.

- In the network analysis of dialogue (see Figure 2):Nodes represent actions made either by a student, teacher, both or neither.

- Shadings of gray and patterns (one checkered and one composed of a grid) represent different types of action (see legend in Figure 2).

- The size of a node is proportional to the time spent on the action the node represents.

- Two nodes are connected if they follow each other in the code sheet.

One way we infer meaning from such mapped dialogues is by summing up the

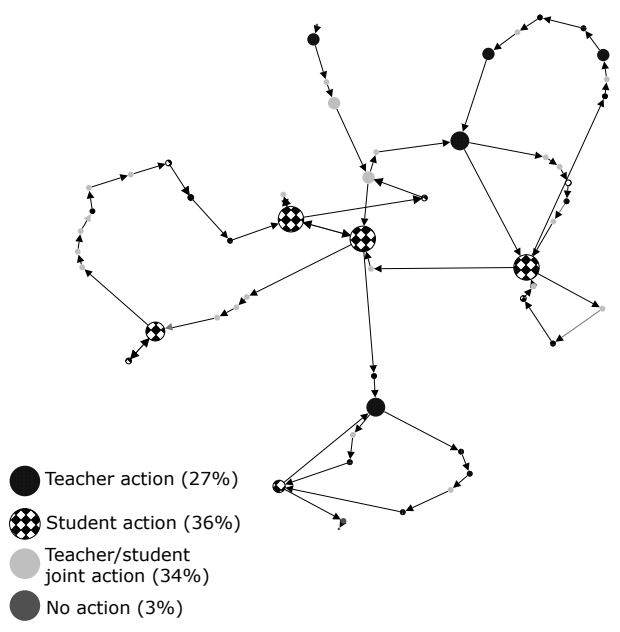

Figure 2 Examples of dialogue mapping where two different teacher-student dialogues were mapped. Case 1 on the left and Case 2 on the right. See text for description of the objects. 
size of all nodes related to each category (student action, teacher action, teacher/ student joint action, and no action) in order to characterise the actions. In Case 1, the teacher is the most prominent category, signifying the dialogue is characterized by the teacher talking (52\%, see Figure 2 ), whereas in Case 2, the student is most prominent (36\%), closely followed by the student-teacher conversations (34\%). These observations may signify that the Case 2 dialogue gave room for the student to express him/herself and that it was actually a dialogue.

Further inferences about Case 2 seem to show that there were 3-4 places (the largest 'student action' nodes) where the student was allowed to express his/her competencies. However, Case 1 seems to show that primarily there was only one place where this happened. This conjecture is further verified by using other codes and a qualitative analysis of the actual dialogues. The presence of many teacher/student nodes in Case 2 may signify a larger degree of negotiation between student and teacher than in Case 1. Interestingly, these negotiations (in Case 2) seem to lead to the student taking/being given more time to express him/herself. This might be inferred from the "strings of beads of 'teacher action' 'Teacher/student joint action' nodes and nodes leading up to the larger 'student action' nodes, which represent prolonged student actions.

These maps reveal that for the student in the Case 2 dialogue, there were many more opportunities to assess their own competence with the tasks being discussed.
The interaction of their statements with those of the teacher gave them both direct and inferred opportunities to assess the relevance and value of their competence. This formative assessment is likely to affect both the motivation for learning and the cognitive outcomes from such a classroom interaction (Harrison et al., 2018). Concurrently, the teacher in the Case 2 dialogue gets formative feedback on the progress of their teaching since they can assess its efficacy in 'real-time' making adjustments for individual learners through this highly interactive dialogue. Teachers also may use such dialogues to begin to summatively assess their teaching approach in the given lesson. Such feedback allows teachers to continuously adjust their interactions to maximize the teaching and learning potential of a given strategy.

\section{Analysis of Group Discussions}

The following example shows a novel way of integrating network analysis with qualitative discourse analysis to develop a bottom-up systematic approach to generating themes in data. The case is a student discussion of sustainability, which illustrates how this integration can make the analysis more sensitive to different voices in the discussion. In this section we introduce the use of a community detection algorithm called Infomap (Rosvall \& Bergstrom, 2008). The purpose of such an algorithm is reduce the apparent complexity of nodes and their connections by creating a map that captures essential larger scale structures in the network. The methodology is 


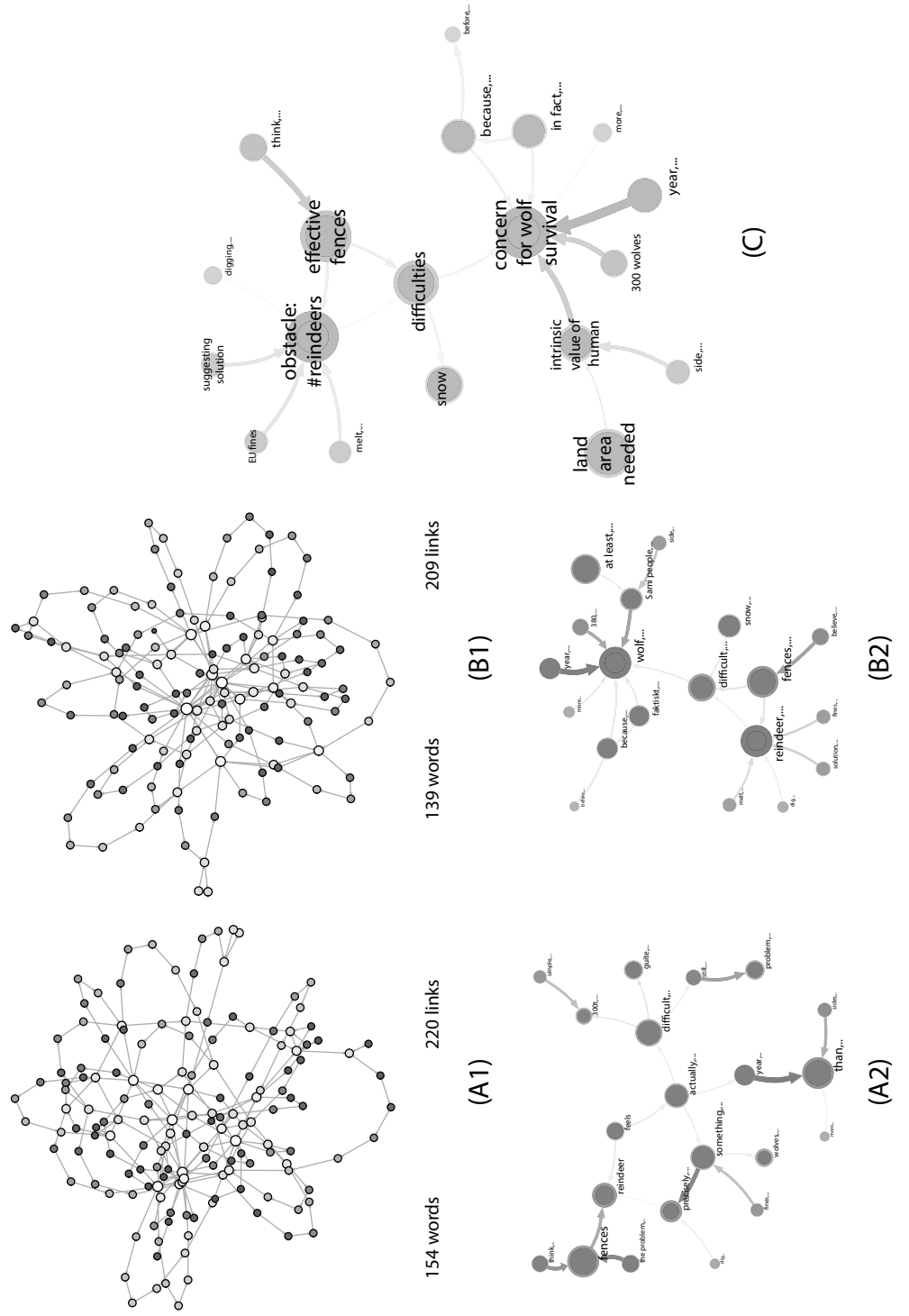

Figure 3 (A1) A network from one of the cycles aligning discourse with network analysis. (A2) The corresponding trial thematic map. (B1) The final network. (B2) The final thematic map. (C) The final thematic map with interpretation. (Adapted from Lindahl et al., 2016.) 
visualized by Lindahl, Bruun, \& Linder (2016) in a poster and is briefly described by Bruun (2016). Here we first describe the context and then provide an overview of the methodology.

As part of a teaching sequence, a group of Swedish students discussed conflicting views presented in a newspaper article about the inbreeding of wolves in Sweden. Initially for this group, the qualitative discourse analysis revealed a discussion that appeared mostly one-sided. It seemed dominated by a single point-of-view (protect the Swedish wolves by using fences). The one-sidedness in the discussion apparently left little room for additional exploration. We now provide a short overview of the methodology that was used to change that interpretation.

Lindahl et al. (2016) presented a methodology that combines qualitative discourse analysis with network analysis - and in particular community detection (see e.g. Barabási, 2016, for an introduction) to extract central themes in a discussion. Bruun, Lindahl, \& Linder (under review) develop the full methodology and describes the empirical details. The method begins with creating a transcript of the discussion. Then qualitative discourse analysis is performed to find units of discourse and eventually themes of the discussion (see e.g. Wood \& Kroger, 2000). Parallel to the qualitative discourse analysis, the original transcript is subjected to standard text mining pre-processing (Feldman \& Sanger, 2007). This entails specific rules for changing the original transcript including removing punctuation, using only lower case letters, and removing common words (for example, the word and in English). Then, a network is created using word adjacency; the remaining words become nodes in a network, and a directed link is created from a word $A$ to a word $B$ if $B$ follows $A$ in the transcript. From this network, a map is created using the Infomap algorithm. This map consists of interconnected modules (A2, B2, and C in Figure 3). Each module has an internal structure of words derived from the original network. The connections between modules stem from words in one module having connections to words in other modules.

Starting from these two initial products, a qualitative discourse analysis and a map based on network analysis, the methodology now requires a process of aligning the two. This happens by carefully scrutinizing the modules in the map and the connections between modules, and comparing them with the qualitative discourse analysis. At first, the two may not match completely. This will likely require new rules for pre-processing. For example, some words might seem redundant and offer little information about the conversation. Another example is synonymous words. If two words are used synonymously in the discussion, one word should be used for both throughout the transcript. In addition, grammatical forms might be reduced to a single form (for example, wolf, wolves, the wolf, the wolves being reduced to just wolf). These choices should be informed by theory and by the research questions one wishes to address. The 
changes that may occur in such a process is illustrated in Figure 3. A1 and A2 shows a network and map based on a transcript that has been subject to standard pre-processing, whereas for B1 and B2 the transcript has been subjected to reductions of the sort just described. The difference can be seen when comparing A2 and B2. The initial map suggests four different parts of the discussion and B2 suggests two overall parts. We attribute the change in this particular case primarily to the grammatical reduction pertaining to wolf. In A2, different grammatical forms of wolf are found in different modules, whereas wolf only appears in one module in B2. Between $\mathrm{A} 1-\mathrm{A} 2$ and $\mathrm{B} 1-\mathrm{B} 2$, there are several intermediate maps and refinements to the interpretations. During this iterative inter- pretative process the qualitative discourse analysis and network analyses are aligned until they produce a combined coherent interpretation. During that process, the modules of the map transform and are at the end seen as themes. The end product is a thematic map (C2 in Figure 3$)$ with an associated qualitative discourse analysis like interpretation.

In the case of the discussion visualized in Figure 3, the interpretations were changed from a one-sided discussion to a tug-of-war system. The final map shows two distinct subsystems - one centered on the theme concern for wolf survival and one with two prominent themes, which has to do with a simple solution to the inbreeding of wolves: effective fences and obstacle: \#reindeers - arising from issues relating to

Table 2 Affordances and challenges of network analysis (Bruun, 2016)

\begin{tabular}{|l|l|}
\hline Things to keep in mind & Explanation \\
\hline What nodes and links represent & $\begin{array}{l}\text { This is an ongoing negotiation, which continues } \\
\text { throughout an analysis. }\end{array}$ \\
\hline Dynamics of networks and on networks & $\begin{array}{l}\text { It is possible to analyse how networks change } \\
\text { over time but also possible to analyse processes } \\
\text { within networks }\end{array}$ \\
\hline $\begin{array}{l}\text { Network visualizations may foster important } \\
\text { questions }\end{array}$ & $\begin{array}{l}\text { Visualizations can guide a researcher to ask } \\
\text { questions. However, different visualizations of } \\
\text { the same data set may foster different questions }\end{array}$ \\
\hline Networks as mathematical objects & $\begin{array}{l}\text { The mathematical affordances of networks } \\
\text { makes them amenable to calculations. However, } \\
\text { every calculation relies on an underlying model } \\
\text { and so that must always be take into account. }\end{array}$ \\
\hline Network science is evolving at a rapid pace & $\begin{array}{l}\text { New ways of analysing networks appear } \\
\text { frequently in the field of network science. This } \\
\text { provides for a plethora of possibilities for using } \\
\text { network analysis. }\end{array}$ \\
\hline
\end{tabular}


effective fences and the number of reindeers. Further scrutiny of the transcripts with this interpretation revealed that the tug-of-war was between obstacles, being more concrete, and intrinsic values, being more abstract.

\section{Networks as a Methodological Tool}

The three illustrations above have all shown different ways of conceptualizing nodes and links as educationally relevant entities. We have shown how one can analyse networks as they evolve over time - the dynamics of networks - and how one can model dynamic processes on networks. We have shown that networks have both visual and mathematical properties that make them tractable as a way of generating knowledge about relational data. We summarize our key points in Table 2.

These things underpin any theory that we might develop from using networks. It will be a theory that emphasises the relational aspects. For example, concerning the social network, we try to generate the theory-like structures rules of interaction, which are meant to have explanatory power. Likewise, the interpretations we make of the student discussions are shaped in part by the algorithm we use to create discussion maps as well as by the changes we make to the transcript. In the teacher-student dialogue, the structure of the network shapes the way we comprehend and talk about the discussion networks. All these are dependent on the relational character of networks and would not be the same without that perspective. In other words, the theoretical positions we can develop are shaped by the nature of networks.

In each of the examples given above, network methodology has been coupled with an appropriate theoretical basis. In the first example, we can extend the analysis by adding a theoretical layer. From the perspective of communities of practices (Wenger, 1998), an argument could be made to not perceive the class as a single community of practice when they do laboratory exercises. While they may negotiate meaning in relatively closed groups, if they do not share their experiences with others, how would they develop a shared repertoire in physics? Of course, physics is more than just solving problems, but we see the same patterns in networks pertaining to conceptual discussions (Bruun, 2012). We find it much more likely that a community of practice could have formed during Week 45, where students prepared for a test. This is based on the visualizations and the calculations of target entropy. If these hypotheses are valid (and they should be tested in other ways as well), then network analysis can be used to elaborate more on how communities of practice may form and live in response to teaching. For example for laboratory work, they seem to be small and formed only for the duration of the laboratory work.

In the second example, network maps of classroom dialogues were used to graphically illustrate for a teacher the nature of their interactions with students and consequently help them adjust their teaching methods based on concrete rep- 
resentations rather than impressionistically remembered versions. When combined with access to a video record of the same teaching episodes, teachers can more accurately sort out the actual structure of their teaching with the possibility of altering it. Concomitantly, education researchers can use dialogue mapping to reveal the structure of student-teacher interactions as a way to gain a fine-grained view of a teaching-learning interaction. When combined with recordings of the same dialogues, researchers can identify factors contributing to the teachinglearning milieu.
In the case of the third example, we coupled networks with qualitative discourse analysis as a methodological tool to build a grounded theoretical understanding of the discussion. We showed that researchers' interpretations of discussions can undergo important changes by iteratively aligning network analysis with qualitative discourse analysis. Doing this for a variety of group discussions may help both researchers and teachers understand the very complex dynamics that can underpin such group discussions. It may help identify group discussions that are conducive for student achievement of particular learning goals.

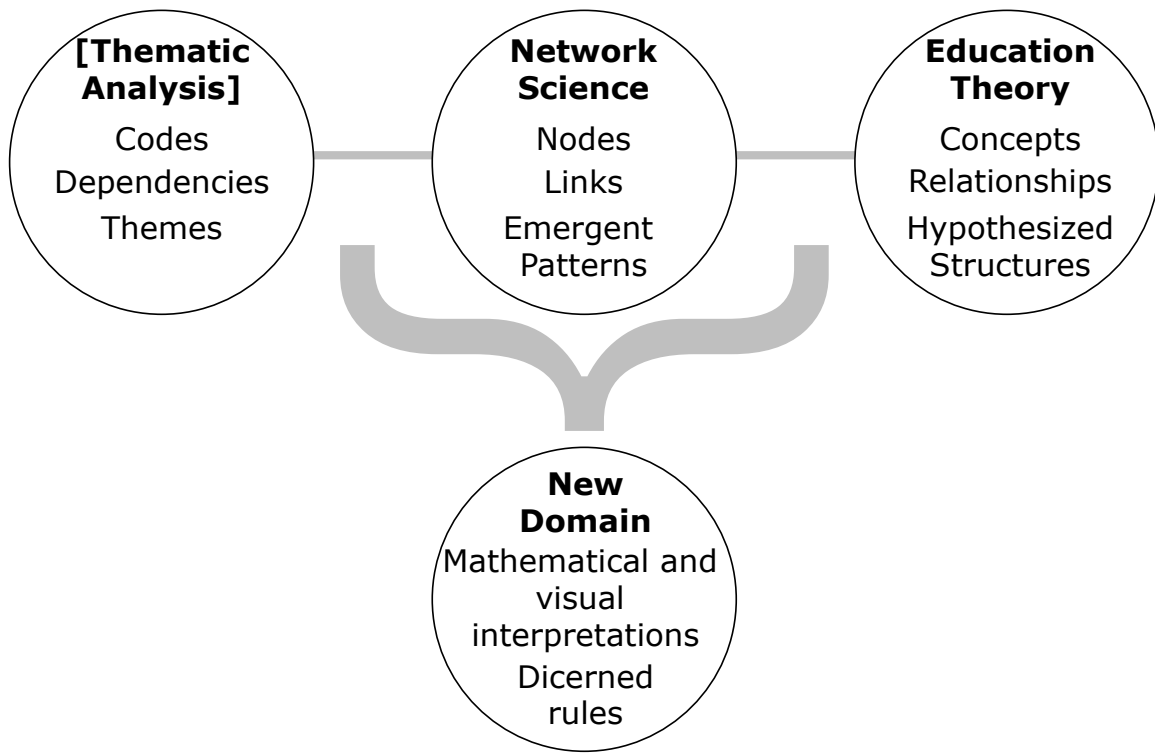

Figure 4 An illustration of how parts of methodological frameworks (here exemplified by Thematic Analysis - Braun \& Clarke, 2006), network science, and educational theory may blend into a new domain of mathematical and visual interpretations. The new domain also contains hypothesized rules about how the emergent patterns visible in the network arose. (From Bruun, 2016.) 
Table 3 Some of the possible properties of the blends made in the three examples

\begin{tabular}{|l|l|l|l|}
\hline Framework & Nodes & Links & Emergent patterns \\
\hline $\begin{array}{l}\text { Communities of } \\
\text { Practice }\end{array}$ & $\begin{array}{l}\text { Students as } \\
\text { structurally embedded } \\
\text { entities }\end{array}$ & $\begin{array}{l}\text { Engagement as a form } \\
\text { of interaction }\end{array}$ & $\begin{array}{l}\text { Are products of rules } \\
\text { of interaction }\end{array}$ \\
\hline Dialogical teaching & $\begin{array}{l}\text { Dialogical interactions } \\
\text { as teaching/learning } \\
\text { opportunities }\end{array}$ & $\begin{array}{l}\text { Flow of actions as } \\
\text { sequential indicators } \\
\text { of process }\end{array}$ & $\begin{array}{l}\text { Are the dialogical } \\
\text { structures, which } \\
\text { become elucidated }\end{array}$ \\
\hline Qualitative analysis & $\begin{array}{l}\text { Words/phrases as } \\
\text { components of } \\
\text { discourse }\end{array}$ & $\begin{array}{l}\text { Flow of discussion } \\
\text { as constitution of } \\
\text { discourse }\end{array}$ & $\begin{array}{l}\text { Are interpreted as } \\
\text { interconnected themes }\end{array}$ \\
\hline
\end{tabular}

The idea behind each of these examples is that of a conceptual blend (Fauconnier \& Turner, 2002; Podolefsky \& Finkelstein, 2007; Bruun, 2012). In short, a conceptual blend consists of at least two domains that are then mapped into a new domain. Part of this can be achieved by seeing the two domains as analogues and then matching concepts in one domain to concepts in the other. For example, mapping nodes from networks to people (students) in communities of practice, and links to engagement. But by doing this, we change the meaning of nodes, the meaning of links and - importantly - also the meaning of student engagement, even of communities of practice. Fauconnier and Turner (2002) argue that we create a new domain where some things in the old domains are left out and things appear that were not part of either of the old domains. Figure 4 illustrates this. Here, we have included Thematic Analysis (Braun \& Clarke, 2006) as an example of a methodological framework that can also go into the blend. In the top row of Figure 4, words have been placed in corresponding places to indicate an analogy. For example, codes in thematic analysis are seen as analogous to nodes in a network that are analogous to concepts in some educational theory. In the blend, these analogies become mathematical and visual entities with accompanying interpretations - here labeled mathematical and visual interpretations. Parts of the new domain are rules that are discerned from the visual and mathematical interpretations. These are to be viewed as underlying rules that are hypothesized to shape the emergent patterns of the network. For example, possible teacher choices in the structured assessment dialogue may be discerned from her patterns of questions and from the student's reactions.

As a first step in characterising some of the new domains that appear when blending network analysis with other frameworks, we have matched nodes, links, and what we call emergent patterns to each framework. We see an emergent pattern in a network as a structure that is derives from nodes and being connected in a network. Thus, interconnected themes in the third example are an emergent structure. 
In the social network example, we have emphasized the rules of interaction as a specific product of the blend that is not part of either network theory or communities of practice, but can certainly be seen as rooted in both. The patterns that we see emerge in a network (e.g. the grouping of male students or the variation in target entropy) are then seen as products of rules of interaction.

\section{FINAL DISCUSSION}

Network analysis is still a new approach in educational research, and we believe that in time it will provide valuable insights into how learning occurs in many different contexts and at many different levels. However, in many ways, the structural and relational views, which are essential for network analyses are new to education. Thus, to realize the potentials of network analysis in education, some developments need to occur.

The first development relates to software. Network analysis requires software tools for visualization and mathematical treatment, and researchers need to become familiar with using and understanding these tools and their limitations. In our analyses, we have made use of free software, Gephi (Bastian et al., 2009), iGraph (Csardi \& Nepusz, 2006) for the $\mathrm{R}$ statistical environment ( $\mathrm{R}$ Core Team, 2016), and Mapequation.org (Bohlin et al., 2014). Many other alternatives exist, and researchers can start their search on Wikipedia (Social network analysis software, 2018). Networks are mathematical objects on which one can perform rich and varied calculations in order to tease out structural patterns, and we have found that no single tool is capable of meeting all our needs. Thus, researchers who want to work network analysis for education will need to learn multiple software tools to achieve insights.

The second development relates to quantitative aspects. Network analysis will usually not produce data, which follow normal distributions. On the contrary, many distributions in network analysis follow power-law or more exotic distributions (Barabási, 2016). This means that standard statistical ideas such as mean value and standard deviation cannot be imbued with the traditional meanings. Thus, extra care needs to be taken when making inferences based on statistics in network analysis. This has implications, for example, when comparing networks. In the first example, we could not compare networks based directly on their target entropy but had to first compare the target entropy of each network with random expectation. However, random expectation can mean many things, and we have opted for a particular model. Depending on the research question, one could imagine different models being relevant. Researchers wishing to utilize the quantitative aspects of network analysis cannot rely solely on the use of standard statistical methods.

The third development relates to combining network analysis with qualitative work. Visualizing networks may aid researchers in different parts of qualitative research. It can make theoretical choices 
made in qualitative discourse analysis clear by showing emergent relations between developing concepts. Networks can also visually aid holistic interpretations of educational data by showing the structural relationships between, for example, developed categories (see e.g. Shaffer et al., 2009). However, this pre-supposes that researchers adopt the structural view of relations that networks require. Converting, for example, a text to a network is a compression, and information will be lost as we gain a bird's eye view of the text. Interpreting the bird's eye view without sufficient knowledge of the text could lead to misinterpretations. Thus, while using network analysis in combination with qualitative analyses will likely yield new possible insights, great care should be made when making interpretations based on the networks.

\section{References}

Artigue, M., \& Winsløw, C. (2010). International comparative studies on mathematics education: A viewpoint from the anthropological theory of didactics. Recherches en didactique des mathématiques, 30(1), 47-82.

Barabási, A. L. (2016). Network science. Cambridge University Press.

Bastian, M., Heymann, S., \& Jacomy, M. (2009). Gephi: an open source software for exploring and manipulating networks. ICWSM, 8, 361-362.

Bohlin, L., Edler, D., Lancichinetti, A., \& Rosvall, M. (2014). Community detection and visualization of networks with the map equation framework. In Y. Dink, R. Rousseau, \& D. Wolfram (Eds.), Measuring Scholarly Impact (pp. 3-34). Cham: Springer.

Braun, V., \& Clarke, V. (2006). Using thematic analysis in psychology. Qualitative Research in Psychology, 3(2), 77-101.

Brewe, E. (2008). Modeling theory applied: Modeling instruction in introductory physics. American Journal of Physics, 76(12), 1155-1160.

Brewe, E., Kramer, L., \& Sawtelle, V. (2012). Investigating student communities with network analysis of interactions in a physics learning center. Physical Review Special Topics - Physics Education Research, 8(1), 010101.

Bruun, J. (2012). Networks in Physics Education Research: A Theoretical, Methodological, and Didactical Explorative Study (Doctoral dissertation). Department of Science Education, University of Copenhagen.

Bruun, J. (2016). Networks as integrated in research methodologies in PER. In D. Jones, L. Ding, \& A. Traxler (Eds.), 2016 PERC Proceedings (pp. 11-17). Sacramento, CA:American Association of Physics Teachers.

Bruun, J., \& Brewe, E. (2013). Talking and learning physics: Predicting future grades from network measures and Force Concept Inventory pretest scores. Physical Review Special Topics - Physics Education Research, 9(2), 020109.

Bruun, J., \& Bearden, I. G. (2014). Time development in the early history of social networks: Link stabilization, group dynamics, and segregation. PLoS ONE, 9(11), e112775. 
Buchenroth-Martin, C., DiMartino, T., \& Martin, A. P. (2017). Measuring student interactions using networks: Insights into the learning community of a large active learning course. Journal of College Science Teaching, 46(3), 90.

Clement, J. (1993). Using bridging analogies and anchoring intuitions to deal with students' preconceptions in physics. Journal of Research in Science Teaching, 30(10), 1241-1257.

Csardi, G., \& Nepusz, T. (2006). The igraph software package for complex network research. Complex Systems, 1695(5), 1-9.

Daly, A. J. (Ed.). (2010). Social network theory and educational change (Vol. 8). Cambridge, MA: Harvard Education Press.

Davis, B., \& Sumara, D. (2006). Complexity and education. New York: Routledge.

Deci, E. L., \& Ryan, R. M. (2011). Self-determination theory. Handbook of Theories of Social Psychology, 1(2011), 416-433.

diSessa, A. A. (2002). Why "conceptual ecology" is a good idea. In Reconsidering conceptual change: Issues in theory and practice (pp. 28-60). Dordrecht: Springer.

Dolin, J., Bruun, J., Nielsen, S. S., Jensen, S. B., \& Nieminen, P. (2018). The structured assessment dialogue. In J. Dolin \& R. Evans (Eds.), Transforming Assessment (pp. 109-140). Cham: Springer.

Fauconnier, G., \& Turner, M. (2002). The way we think: Conceptual blending and the mind's bidden complexities. New York: Basic Books.

Feldman, R., \& Sanger, J. (2007). The text mining handbook: advanced approaches in analyzing unstructured data. Cambridge University Press.

Forsman, J., Moll, R., \& Linder, C. (2014). Extending the theoretical framing for physics education research: An illustrative application of complexity science. Physical Review Special Topics-Physics Education Research, 10(2), 020122.

Goertzen, R. M., Brewe, E., \& Kramer, L. (2013). Expanded markers of success in introductory university physics. International Journal of Science Education, 35(2), 262-288.

Grunspan, D. Z., Wiggins, B. L., \& Goodreau, S. M. (2014). Understanding classrooms through social network analysis: A primer for social network analysis in education research. CBE-Life Sciences Education, 13(2), 167-178.

Gregorcic, B., Planinsic, G., \& Etkina, E. (2017). Doing science by waving hands: Talk, symbiotic gesture, and interaction with digital content as resources in student inquiry. Physical Review Physics Education Research, 13(2), 020104.

Harrison, C., Constantinou, C. P., Correia, C. F., Grangeat, M., Hähkiöniemi, M., Livitzis, M., ... Viiri, J. (2018). Assessment on-the-fly: Promoting and collecting evidence of learning through dialogue. Transforming Assessment (pp. 83-107). Cham: Springer.

Lemke, J. L. (1990). Talking science: Language, learning, and values. Norwood: Ablex.

Lijnse, P. \& Klaassen, K. (2004). Didactical structures as an outcome of research on teaching-learning sequences? International Journal of Science Education, 26(5), 537-554. 
Lindahl, M., Bruun, J., \& Linder, C. (2016). Integrating text-mining, network analysis and thematic discourse analysis to produce maps of student discussions about sustainability. In PERC 2016.

Maslov, S., Sneppen, K., \& Zaliznyak, A. (2004). Detection of topological patterns in complex networks: correlation profile of the internet. Physica A: Statistical Mechanics and its Applications, 333, 529-540.

Masucci, A. P., \& Rodgers, G. J. (2006). Network properties of written human language. Physical Review E, 74(2), 026102.

Macfadyen, L. P., \& Dawson, S. (2010). Mining LMS data to develop an "early warning system" for educators: A proof of concept. Computers \& education, 54(2), 588-599.

Podolefsky, N. S., \& Finkelstein, N. D. (2007). Analogical scaffolding and the learning of abstract ideas in physics: An example from electromagnetic waves. Physical Review Special Topics - Physics Education Research, 3(1), 010109.

$\mathrm{R}$ Core Team (2016). R: A language and environment for statistical computing. Retrieved form www.R-project.org/

Rosvall, M., \& Bergstrom, C. T. (2008). Maps of random walks on complex networks reveal community structure. Proceedings of the National Academy of Sciences, 105(4), 1118-1123.

Shaffer, D. W., Hatfield, D., Svarovsky, G. N., Nash, P., Nulty, A., Bagley, E., ... \& Mislevy, R. (2009). Epistemic network analysis: A prototype for 21st-century assessment of learning. International Journal of Learning and Media, 1(2), 33-53.

Sneppen, K., Trusina, A., \& Rosvall, M. (2005). Hide-and-seek on complex networks. EPL (Europhysics Letters), 69(5), 853.

Social network analysis software (n.d.). In Wikipedia. Retrieved February 7, 2018, from https://en.wikipedia.org/wiki/Social_network_analysis_software

Wenger, E. (1998). Communities of practice: Learning, meaning, and identity. Cambridge University Press.

Wood, L. A., \& Kroger, R. O. (2000). Doing discourse analysis: Methods for studying action in talk and text. Thousand Oaks, CA: Sage.

Assistant professor Jesper Bruun, PhD

Department of Science Education, University of Copenhagen, Denmark; e-mail: jbruun@ind.ku.dk

Associate professor Robert Evans

Department of Science Education, University of Copenhagen, Denmark; e-mail: evans@ind.ku.dk 